\title{
Short-lived and Long-lived Outbursts in B and Be Stars from Hipparcos Photometry and Modelling
}

\author{
A. M. Hubert ${ }^{1}$, M. Floquet ${ }^{1}$, J. Zorec $^{2}$ \\ ${ }^{1}$ Dasgal, UMR8633 du CNRS, Observatoire de Paris-Meudon, F-92195 \\ Meudon, France \\ ${ }^{2}$ Institut d'Astrophysique de Paris - CNRS, 98bis Bd Arago, \\ 75014-Paris, France
}

\begin{abstract}
The number of Be stars which showed short- and long-lived outbursts during the Hipparcos photometric survey, previously reported in Hubert and Floquet (1998), have been increased by examination of light curves of fainter objects. Long-lived outbursts have been modelled as being due to an ejected layer/slab which becomes gradually diluted.
\end{abstract}

\section{Introduction}

Ground-based intensive photometric campaigns on several targets and long-time photometric surveys have allowed to detect outbursts in Be stars. Using Hipparcos photometry Hubert \& Floquet (1998) have extended the outburst detection to a very large number of $\mathrm{Be}$ stars, some of them having been poorly observed in photometry and/or in spectroscopy up to now; outbursts have been preferentially detected with a higher level $(\Delta \mathrm{Hp} \leq 0.3 \mathrm{mag})$ in early Be stars with a low to moderate $v \sin i$. Pavlovski et al. (1998) derived the same conclusion from their investigation of Hvar survey data (Pavlovski et al. 1997).

\section{Investigation of Hipparcos Variability Annex}

In Hubert \& Floquet (1998) the search for the presence of outbursts was first made for bright Be stars $(\mathrm{V} \leq 7)$ selected from the list established by Jaschek $\&$ Egret (1982). In a second step unsolved light curves reported in Hipparcos Variability Annex (part C) for stars with $\mathrm{V}<10$ were investigated to enlarge the number of detections of Be stars which showed during the Hipparcos mission (from mid-1989 to mid-1993) short-lived (days, tens of days), sometimes recurrent, outbursts or/and long-lived outbursts. These later are characterized by a strong increase of brightness $(\Delta \mathrm{Hp} \leq 0.3 \mathrm{mag})$ over a hundred or several hundred days followed by a slow gradual decrease.

Using the list of Be stars given by Jaschek \& Egret (1982), a recent study on newly discovered Be stars and $v \sin i$ measurements by Halbedel (1996), Slettebak (1982) and the SIMBAD database, we have found that in fact the B stars whose unsolved light curves are reported in the Hipparcos catalog are mainly Be stars. 
Table 1. Short-lived outbursts from Hipparcos photometry

\begin{tabular}{|c|c|c|c|c|c|c|}
\hline HD & Name & Sp. Type & $\underset{d}{\text { Time span }}$ & $\begin{array}{l}\Delta \mathrm{Hp} \\
\text { mag }\end{array}$ & $\begin{array}{l}v \sin i \\
\mathrm{~km} / \mathrm{s}\end{array}$ & Remarks \\
\hline 6226 & & B2 IV-V & 230 & 0.08 & & (1) \\
\hline 11606 & V777 Cas & $\mathrm{B} 1 \mathrm{Ve}$ & $50-100$ & 0.15 & & (2) \\
\hline 13051 & V351 Per & B1 IV & $400 ?$ & 0.14 & 168 & \\
\hline 15450 & V555 Per & B1 IIIe & & 0.17 & & \\
\hline 25348 & DE Cam & $\mathrm{B} 1 \mathrm{Ve}$ & & 0.12 & & (3) \\
\hline 25734 & & $\mathrm{Bg}$ & & 0.12 & & \\
\hline 33152 & V413 Aur & $\mathrm{B} 1 \mathrm{Ve}$ & $500 / 200$ & 0.23 & & (4) \\
\hline 33328 & $\lambda$ Eri & $\mathrm{B} 1 \mathrm{Ve}$ & $50-100$ & 0.08 & 220 & (2) \\
\hline 37490 & $\omega$ Ori & B3 IIIe & $250-300$ & 0.06 & 160 & (2) \\
\hline 37657 & V434 Aur & $\mathrm{B3} \mathrm{Ve}$ & $200-300$ & 0.15 & & (2) \\
\hline 40978 & V447 Aur & $\mathrm{B} 1 \mathrm{Ve}$ & & 0.14 & 200 & \\
\hline 46547 & & $\mathrm{~B} 2 \mathrm{~V}$ & & 0.06 & 120 & (5) \\
\hline 50868 & V744 Mon & $\mathrm{B} 2 \mathrm{Ve}$ & & 0.2 & & \\
\hline 56139 & $\omega \mathrm{CMa}$ & B2 IV-Ve & $200 / 330$ & 0.30 & 80 & (2) \\
\hline 62753 & V387 Pup & $\mathrm{B3} \mathrm{Ve}$ & & 0.11 & & (4) \\
\hline 67888 & PQ Pup & $\mathrm{B3} \mathrm{Ve}$ & $100-200$ & 0.20 & & (2) \\
\hline 68570 & V426 Pup & B2 IIIe & $200 / 250$ & 0.14 & & \\
\hline & DO Cru & $\mathrm{B} 2 \mathrm{Ve}$ & & 0.21 & & (3) \\
\hline 102776 & & B3 Ve & & 0.08 & 210 & \\
\hline 105521 & V817 Cen & B3 IVe & $50-100$ & 0.17 & 130 & (2) \\
\hline 120324 & $\mu$ Cen & B2 IV-Ve & $20-30$ & 0.06 & 155 & (2) \\
\hline 120991 & V767 Cen & B2 IIIe & $250-330 ?$ & 0.25 & 70 & (2) \\
\hline 127449 & V1008 Cen & $\mathrm{B} 2 / 3 \mathrm{Ve}$ & & 0.08 & & \\
\hline 128293 & CK Cir & $\mathrm{B3} \mathrm{Ve}$ & & 0.08 & & \\
\hline 164284 & 66 Oph & $\mathrm{B} 2 \mathrm{Ve}$ & $400-500$ & 0.15 & 240 & (2) \\
\hline 174513 & V457 Sct & $\mathrm{B} 1 \mathrm{Ve}$ & & 0.25 & 200 & \\
\hline 180126 & V1448 Aql & B3e & & 0.17 & & \\
\hline 186272 & V341 Sge & $\mathrm{B} 2.5 \mathrm{Ve}$ & & 0.17 & & \\
\hline 187811 & V395 Vul & $\mathrm{B} 2.5 \mathrm{Ve}$ & $100-200$ & 0.11 & 230 & (2) \\
\hline 193009 & V2113 Cyg & $\mathrm{B} 1 \mathrm{Ve}$ & $100-300$ & $0.18-0.20$ & & (2) \\
\hline 194335 & V2119 Cyg & $\mathrm{B} 2 \mathrm{Ve}$ & $150 ?$ & 0.10 & 350 & (2) \\
\hline 194883 & V2120 Cyg & $\mathrm{B} 2 \mathrm{Ve}$ & $100-200$ & 0.20 & & (2) \\
\hline 195907 & V2123 Cyg & B1.5 Ve & $200 / 300$ & 0.14 & & \\
\hline 197419 & V568 Cyg & B2 IV-Ve & & 0.15 & & \\
\hline 204722 & V2162 Cyg & $\mathrm{B} 2 \mathrm{Ve}$ & & 0.10 & & (6) \\
\hline 211835 & V404 Lac & $\mathrm{B3} \mathrm{Ve}$ & $200 / 300$ & 0.2 & & (7) \\
\hline 212076 & $31 \mathrm{Peg}$ & $\mathrm{B} 1 \mathrm{Ve}$ & & 0.30 & 100 & \\
\hline 223036 & V817 Cas & $\mathrm{Be}$ & $100 / 700$ & 0.305 & & \\
\hline
\end{tabular}

(1) suspected to be a Be star by Božić \& Harmanec (1998, A\&A330, 222)

(2) already reported in Hubert \& Floquet (1998)

(3) with long-term variations

(4) few data, several outbursts or quasi-periodic oscillations (QPO)

(5) star in a triple system

(6) with a slow decrease

(7) with long-term variations or long-lived outbursts?

- $38 \mathrm{~B}$ and Be stars which showed short-lived outbursts are listed in Table 1; all of them except two stars have a spectral type earlier than B4. In some cases time span between short-lived outbursts could be estimated and maximum intensity associated with them. In spite of the lack of $v \sin i$ determination for fainter ones, stars with short-lived outbursts are seen with a low to moderate inclination except in one case. 
- $32 \mathrm{~B}$ and Be stars which showed long-lived outbursts are listed in Table 2. Spectral types are mainly earlier than B3. Stars having a vsini determination are found with a moderate inclination angle. An estimation of the duration of brightness increase and of the subsequent decay is also given in Table 2.

Table 2. Long-lived outbursts from Hipparcos photometry.

\begin{tabular}{|c|c|c|c|c|c|c|c|}
\hline HD & Name & Sp. Type & $\begin{array}{l}\text { Increase } \\
\text { d }\end{array}$ & $\begin{array}{l}\text { Decrease } \\
\mathrm{d}\end{array}$ & $\begin{array}{l}\Delta \mathrm{Hp} \\
\mathrm{mag}\end{array}$ & $\begin{array}{r}v \sin i \\
\mathrm{~km} / \mathrm{s}\end{array}$ & Remarks \\
\hline & V399 Cep & B2 & 150 & $200 / 300$ & 0.192 & & \\
\hline 20809 & V 575 Per & B5 V & $\leq 200$ & $\begin{array}{l}\sim 600 \\
\sim 200\end{array}$ & 0.049 & 250 & (1) \\
\hline & CT Cam & B2 Ve & & $\geq 600$ ? & 0.132 & & \\
\hline 29441 & V1150 Tau & B2 Ve & $<150$ & $>400$ & 0.097 & & (2) \\
\hline 34921 & V420 Aur & $\mathrm{B} 0 \mathrm{rVe}$ & 300 & $>500$ & 0.111 & 240 & \\
\hline 34986 & V423 Aur & B8 & 180 & 400 & 0.158 & & (3) \\
\hline 36012 & V1372 Ori & B5 Ve & $<200$ & $>800$ & $\geq 0.18$ & & (4) \\
\hline 49131 & $\mathrm{HP} \mathrm{CMa}$ & $\mathrm{B} 2 \mathrm{Ve}$ & 100 & 400 & $\overline{0} .35$ & & $(4,5)$ \\
\hline & LL CMa & $\mathrm{B} 3 \mathrm{Ve}$ & $\leq 150$ & $\geq 300$ & 0.276 & 150 & \\
\hline 53085 & V749 Mon & B8e & $\sim 250$ & 550 & 0.167 & & \\
\hline 53367 & V750 Mon & Bo IVe & $\sim 400$ & & 0.25 & & (4) \\
\hline 54309 & FV CMa & $\mathrm{B} 2 \mathrm{Ve}$ & 100 & $>400$ & 0.20 & 200 & (4) \\
\hline 55538 & $\mathrm{HI} \mathrm{CMa}$ & B2 Ve & 200 & 250 & 0.214 & 130 & \\
\hline 57539 & V757 Mon & B5 III & $\geq 300$ ? & & 0.091 & & \\
\hline 92938 & V518 Car & B3 Ve & 200 & 500 & 0.15 & & (4) \\
\hline 306798 & V911 Cen & $\mathrm{Be}$ & $\sim 100$ & $\sim 300$ & 0.447 & & (6) \\
\hline & V959 Cen & $\mathrm{Be}$ & 200 & 350 & 0.335 & & \\
\hline 106881 & KV Mus & B3 Ve & 130 & 300 & 0.151 & & \\
\hline 127489 & V1010 Cen & $\mathrm{B} 2 \mathrm{Ve}$ & $\sim 200$ & $\sim 400$ & 0.300 & & \\
\hline 130287 & CP Cir & B5 IVe & $\sim 170$ & $\sim 350$ & 0.228 & & \\
\hline 148259 & OZ Nor & $\mathrm{B} 2 \mathrm{Ve}$ & $\sim 100 ?$ & 200 & 0.290 & & \\
\hline 171406 & V532 Lyr & $\mathrm{B} 4 \mathrm{Ve}$ & & $\geq 350$ & 0.094 & & \\
\hline 178175 & V4024 Sgr & B2 Ve & $\geq 300$ & & $\geq 0.12$ & 120 & (4) \\
\hline 198895 & V417 Cep & $\mathrm{B} 1 \mathrm{Ve}$ & $\overline{100}$ & 200 & 0.194 & 190 & \\
\hline 200269 & V2144 Cyg & B5 Ve & $250 ?$ & 400 & 0.150 & & \\
\hline 202904 & $v$ Cyg & $\mathrm{B} 2 \mathrm{Ve}$ & 100 & $>400$ & 0.20 & 180 & (4) \\
\hline 203467 & $6 \mathrm{Cep}$ & B3 IVe & $<200$ & & $\geq 0.20$ & 150 & (4) \\
\hline 206135 & V433 Cep & $\mathrm{B} 3 \mathrm{Ve}$ & $150 ?$ & 300 & $\overline{0} .170$ & & \\
\hline 211835 & V404 Lac & B3 Ve & $300 ?$ & $500 ?$ & 0.195 & & (6) \\
\hline 213129 & V409 Lac & $\mathrm{B} 2 \mathrm{e}$ & $\sim 250$ & 500 & 0.208 & 190 & \\
\hline 239618 & V420 Cep & $\mathrm{B} 2 \mathrm{Ve}$ & $50 / 100$ & $100 / 200$ & 0.29 & & (7) \\
\hline
\end{tabular}

(1) dimple activity in He I, Smith et al.(1996, ApJ469, 336)

(2) long-lived outburst or quasi-cyclical variation

(3) recurrent long-lived outbursts?

(4) already reported in Hubert \& Floquet (1998)

(5) recurrent long-lived outbursts

(6) several short-lived outbursts prior to a long-lived one

(7) several long-lived outbursts with different intensities

\section{A model for light curves of long-lived outbursts}

We assume that the star ejects a massive layer whose mass is roughly given by: $\Delta M / M_{\odot} \sim 810^{-11}\left(R_{\star} / R_{\odot}\right)^{2} E \tau_{e}$ where $\tau_{e}$ is the initial radial electron scattering opacity of the ejected layer and $E$ is its ellipticity. Two scenarios were 

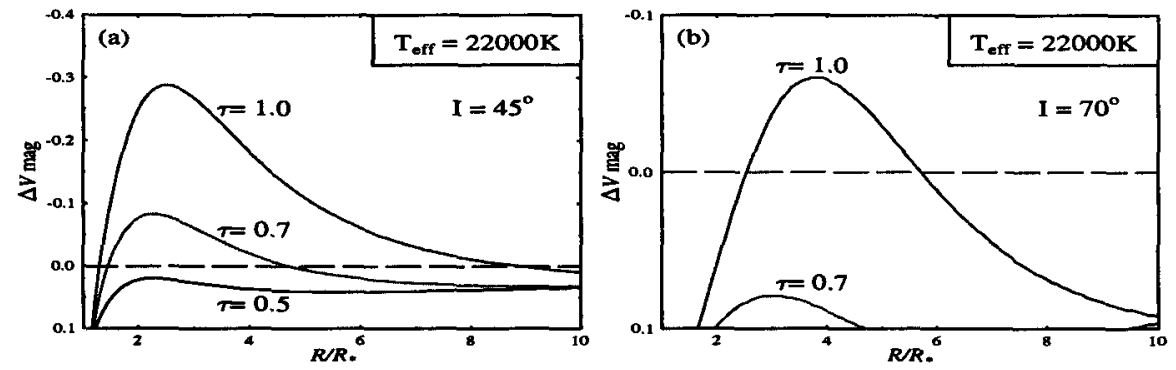

Figure 1. Variation of the magnitude $V$ as a function of the external radius $R / R_{*}$ and of the aspect angle $i$ of an ejected layer whose ellipticity is variable $E=R_{\star} / R$. The ejected mass is roughly: $\Delta M / M_{\odot} \sim 810^{-11}\left(R_{\star} / R_{\odot}\right)^{2} E \tau_{e}$

calculated: (a) once the layer is ejected, it goes on expanding preserving its total mass and its ellipticity $E$; (b) the expansion of the layer pursues preserving its mass but its ellipticity changes as $E=R_{\star} / R$, where $R$ is the equatorial radius of the expanding ellipsoidal layer.

The light change produced by the expanding layer is understood in terms of the well known optical depth variation of the layer (Sobolev 1990). Hence, two phases are distinguished. In the first phase, the opacity is high and the photometric evolution of the star-layer system is determined by the increase of the layer radius $R$. In the second phase, the layer becomes progressively transparent as its opacity decreases. The reduction of its optical depth is due to the lowering of its mean opacity during the expansion. In Fig. 1 are presented the light curves for ejected layers which follow scenario (a). These scenarios may easily explain light curves observed during the Hipparcos mission. The final shapes of these curves still depend on the velocity law assumed for the expansion. On the other hand, we can also see that these massive ejections might supply enough matter to build up circumstellar envelopes in Be stars, as in each ejection there is about as much matter as ejected by an annual continuous mass ejection process. It is expected, however, that the discrete mass ejections will produce clumpy circumstellar envelopes or with quite irregular mass distributions.

\section{References}

Halbedel, E. M. 1996, PASP 108, 833

Hubert, A.M., Floquet, M. 1998, A\&A 335, 565

Jaschek, M., Egret, D. 1982, Publ. Spéc. CDS, 4

Pavlovski, K., Harmanec, P., Božić, H. et al. 1997, A\&AS 125, 75

Pavlovski, K., Ružić Ž., Dominis, D. 1998, in " Cyclical Variability in Stellar Winds", eds. A. Fullerton and L. Kaper, p. 141

Slettebak, A. 1982, ApJS 50, 55 